01,09

\title{
Особенности электронной структуры и спектральных характеристик соединения $\mathrm{Gd}_{5} \mathrm{Si}_{3}$
}

\author{
(C) Ю.В. Князев ${ }^{1}$, А.В. Лукоянов ${ }^{1,2}$, Ю.И. Кузьмин ${ }^{1}$ \\ ${ }^{1}$ Институт ффизики металлов им. М.Н. Михеева УрО РАН, \\ Екатеринбург, Россия \\ ${ }^{2}$ Уральский федееральный университет им. Б.Н. Ельцина, \\ Екатеринбург, Россия \\ E-mail: knyazev@imp.uran.ru
}

(Поступила в Редакцию 13 июля 2016 г.)

\begin{abstract}
Представлены результаты исследований электронной структуры и оптических свойств гексагонального интерметаллического соединения $\mathrm{Gd}_{5} \mathrm{Si}_{3}$. Проведены спин-поляризованные расчеты зонного спектра в приближении локальной электронной плотности с учетом сильных электронных корреляций в $4 f$-оболочке иона Gd (метод LSDA + U). В интервале длин волн $0.22-15 \mu \mathrm{m}$ методом эллипсометрии определены оптические постоянные соединения, рассчитан ряд спектральных характеристик. На основе рассчитанной плотности электронных состояний анализируется поведение частотной зависимости оптической проводимости в области квантового поглощения света.
\end{abstract}

Работа выполнена в рамках государственного задания ФАНО России (тема „Электрон“, № 01201463326) при частичной поддержке РФФИ (проекты № 15-52-45009 и 16-52-48012), проекта 15-8-2-4 Комплексной программы РАН. А.В.Л. благодарит за поддержку Правительство Российской Федерации (постановление № 211, контракт № 02.А03.21.0006) и стипендиальную программу Президента РФ СП-226.2015.2.

DOI: 10.21883/FTT.2017.03.44147.295

\section{1. Введение}

Интерметаллические соединения, представляющие семейство редкоземельных силицидов $\mathrm{R}_{5} \mathrm{Si}_{3}(\mathrm{R}-$ редкоземельный металл $(\mathrm{P} 3 \mathrm{M}))$, характеризуются многообразием электронных и магнитных свойств. Интерес к исследованию бинарных и псевдобинарных соединений такого типа существенно усилился после открытия в системе сплавов $\mathrm{Gd}_{5}\left(\mathrm{Si}_{x} \mathrm{Ge}_{4-x}\right)$ гигантских магнетокалорических, магнитострикционных и магниторезистивных эффектов [1-5]. Большая величина этих эффектов связана с трансформациями в магнитном состоянии, происходящими под влиянием внешнего магнитного поля $[3,6]$, температуры $[3,7]$ или давления $[8,9]$. Результаты экспериментальных исследований показали сильную взаимозависимость магнитных, электронных и структурных свойств таких материалов. Было синтезировано и внимательно изучено большое количество соединений данной серии. Редкоземельные силициды, в которых $\mathrm{R}$ являются тяжелыми лантаноидами, кристаллизуются в гексагональной структуре типа $\mathrm{Mn}_{5} \mathrm{Si}_{3}$. В данной кристаллической решетке атомы РЗМ локализованы в двух неэквивалентных позициях, образующих различные подрешетки, что приводит к анизотропии внутренних магнитных взаимодействий. В результате конкуренции таких взаимодействий происходит формирование различных типов неколлинеарных магнитных структур, существенно влияющих на магнитные и транспортные характеристики соединений $\mathrm{R}_{5} \mathrm{Si}_{3}$. Установлено, что эти характеристики проявляют аномальное поведение под влиянием внешних воздействий [10-16]. Разнообразие физических свойств указанных интерметаллидов стимулирует интерес к исследованию их электронной структуры. Для ряда таких соединений были проведены зонные расчеты энергетического спектра, в которых определена природа электронных состояний вблизи уровня Ферми, а также рассчитаны атомные магнитные моменты [17-19]. Строение внутренних и внешних энергетических зон некоторых силицидов РЗМ исследовалось методами фотоэмиссионной и оптической спектроскопии [18,20,21].

В настоящей работе исследуются электронная структура и оптические характеристики бинарного интерметаллида $\mathrm{Gd}_{5} \mathrm{Si}_{3}$, ряд магнитных, кинетических и структурных свойств которого представлены в работах [19,22-24]. В них сообщается, что в температурном интервале ниже $T_{N} \approx 75 \mathrm{~K}$ соединение находится в антиферромагнитной (АФМ) фазе, характеризуемой геликоидальным упорядочением спиновых моментов. Особенности перехода в АФМ состояние проявляются в аномалиях на температурных зависимостях магнитной восприимчивости и электросопротивления.

\section{2. Расчет электронной структуры}

Соединение $\mathrm{Gd}_{5} \mathrm{Si}_{3}$ кристаллизуется в гексагональной структуре типа $\mathrm{Mn}_{5} \mathrm{Si}_{3}$ (пространственная группа $\left.P 6_{3} / \mathrm{mcm}\right)$. Атомы $\mathrm{Gd}$ в элементарной ячейке занимают две неэквивалентные кристаллографические позиции: $\mathrm{Gd} 1-4 d(1 / 3,2 / 3,0)$ и $\mathrm{Gd} 2$ $6 g\left(x_{\mathrm{Gd}}=0.2402(1), 0,1 / 4\right)$, атомы $\mathrm{Si}$ располагаются в позициях $6 g\left(x_{\mathrm{Si}}=0.6040(2), 0,1 / 4\right)[19]$. 
Расчеты электронной структуры данного соединения проведены в рамках самосогласованного метода LSDA + U [25] в пакете программ TB-LMTO-ASA [26] на основе метода линеаризованных маффин-тин (МТ) орбиталей в приближении атомных сфер. При этом учитывалось сильное кулоновское взаимодействие электронов в $4 f$-оболочке $\mathrm{Gd}$. Численные значения параметров прямого кулоновского $U=6.7 \mathrm{eV}$ и обменного $J=0.7 \mathrm{eV}$ взаимодействий для ионов $\mathrm{Gd}$ соответствуют величинам, используемым в $[27,28]$. В орбитальный базис были включены МТ-орбитали, соответствующие $6 s-, 6 p-, 5 d-$ и $4 f$-состояниям $\mathrm{Gd}$, а также $3 s-, 3 p$ - и $3 d$-состояниям Si. В расчетах использовались следующие радиусы МТ-сфер: для $\mathrm{Gd}$ - 3.6 a.u., $\mathrm{Si}$ - 2.9 a.u. Для заполнения пустого пространства также были добавлены пустые сферы с радиусом 2.2 a. u. Моделировалось АФМ упорядочение локальных магнитных моментов для двух типов ионов $\mathrm{Gd}$, которое по полной энергии оказалось более выгодным, нежели ферромагнитное упорядочение. Рассчитанная величина магнитного момента на ионах гадолиния $\left(7.8 \mu_{\mathrm{B}}\right)$ близка к экспериментальным значениям: $7.5 \mu_{\mathrm{B}}$ [29], 8.2 $\mu_{\mathrm{B}}$ [19], 8.34 $\mu_{\mathrm{B}}$ [30] и $8.5 \mu_{\mathrm{B}}$ [23].

На рис. 1 представлена полная плотность электронных состояний $N(E)$ соединения $\mathrm{Gd}_{5} \mathrm{Si}_{3}$ рассчитанная для энергетических зон с противоположными спиновыми проекциями ( $\uparrow$ и $\downarrow)$. Интенсивные пики в зависимости $N(E)$ формируются узкими $4 f$-зонами гадолиния, которые локализованы ниже уровня Ферми $E_{\mathrm{F}}$ в интервале 8-9eV, а выше $E_{\mathrm{F}}-$ вблизи $2 \mathrm{eV}$. Здесь же показаны парциальные плотности состояний для $4 f$-, $5 d$ и $6 p$-электронов $\mathrm{Gd}$, а также $3 d$ - и $3 p$-электронов $\mathrm{Si}$. Суперпозиция этих вкладов (без $4 f$-зон) формирует широкую многопиковую полосу существенно меньшей интенсивности, расположенную в диапазоне энергий $-6 \mathrm{eV}<E_{\mathrm{F}}<8 \mathrm{eV}$ (рис. 1,c). Обращает внимание почти симметричное расположение максимумов и минимумов в обеих спиновых подсистемах. Вклад от $\mathrm{Gd} 6 s-$ и $\mathrm{Si}$ $3 s$-состояний в полную плотность $N(E)$ мал и размыт почти по всей исследуемой области энергий.

\section{3. Результаты и обсуждение}

Исследованное соединение $\mathrm{Gd}_{5} \mathrm{Si}_{3}$ было получено методом индукционной плавки в атмосфере чистого аргона по методу, описанному в [19]. Данные рентгеноструктурного анализа, проведенного на отожженном образце, подтвердили однофазность гексагональной структуры типа $\mathrm{Mn}_{5} \mathrm{Si}_{3}$. Величины параметров кристаллической решетки, используемых при расчете электронной структуры, близки к значениям, полученным в работе [19].

Оптические свойства соединения исследованы при комнатной температуре в интервале длин волн $\lambda=0.22-15 \mu \mathrm{m}(0.083-5.64 \mathrm{eV})$. Показатели преломления $n(\lambda)$ и коэффициенты поглощения $k(\lambda)$, представленные на рис. 2, измерены эллипсометрическим методом Битти, основанным на определении отношения амплитуд и разностей фаз отраженного света $s$ - и $p$-поляризаций. Зеркальная отражающая поверхность образца получена механической полировкой на алмазных пастах различ-

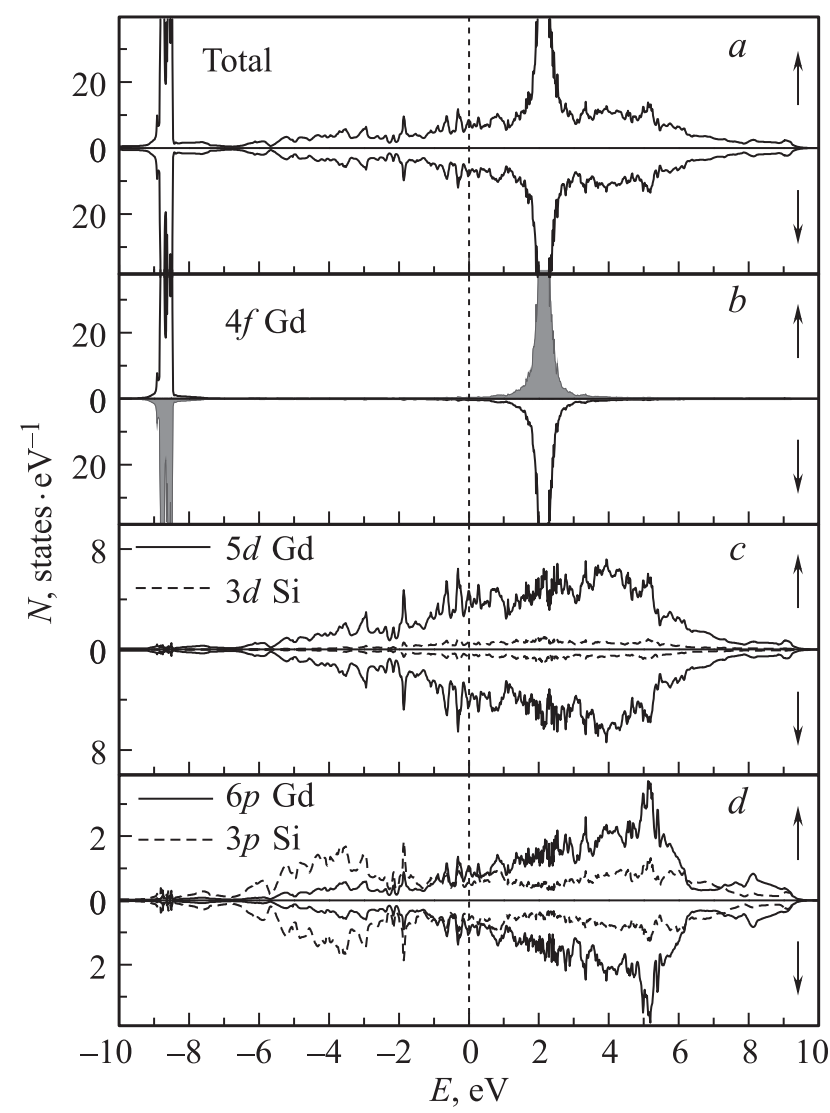

Рис. 1. Плотность электронных состояний соединения $\mathrm{Gd}_{5} \mathrm{Si}_{3}$ : полная $(a)$ и парциальные для $4 f$-электронов $\mathrm{Gd}(b)$ (сплошная линия и затемненные области соответствуют атомам $\mathrm{Gd}$ с противоположно направленными спиновыми моментами), $5 d$-электронов $\mathrm{Gd}$ и $3 d$-электронов $\mathrm{Si}(c), 6 p$-электронов $\mathrm{Gd}$ и $3 p$-электронов $\mathrm{Si}(d)$. Уровень Ферми соответствует нулю на шкале энергий.

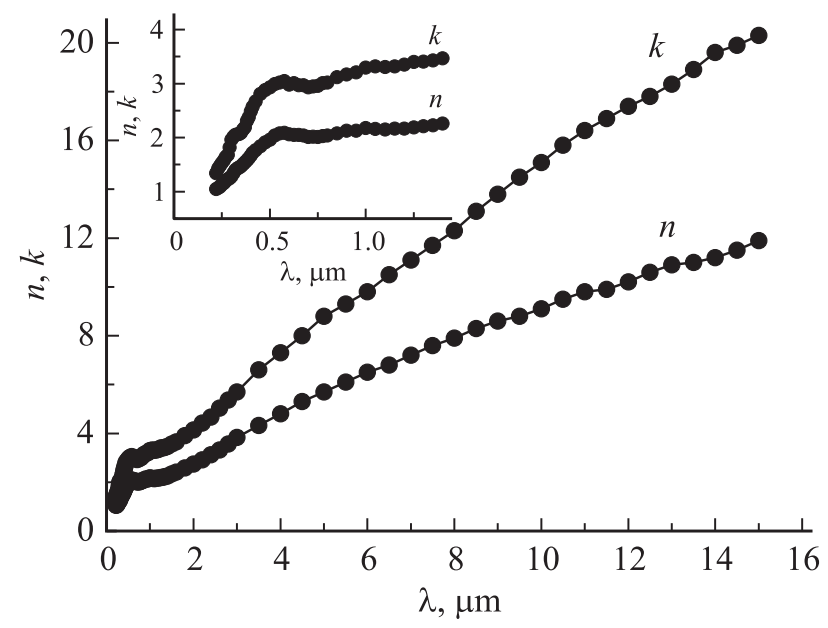

Рис. 2. Зависимость показателя преломления $n$ и коэффициента поглощения $k$ соединения $\mathrm{Gd}_{5} \mathrm{Si}_{3}$ от длины волны. На вставке показан коротковолновый интервал. 

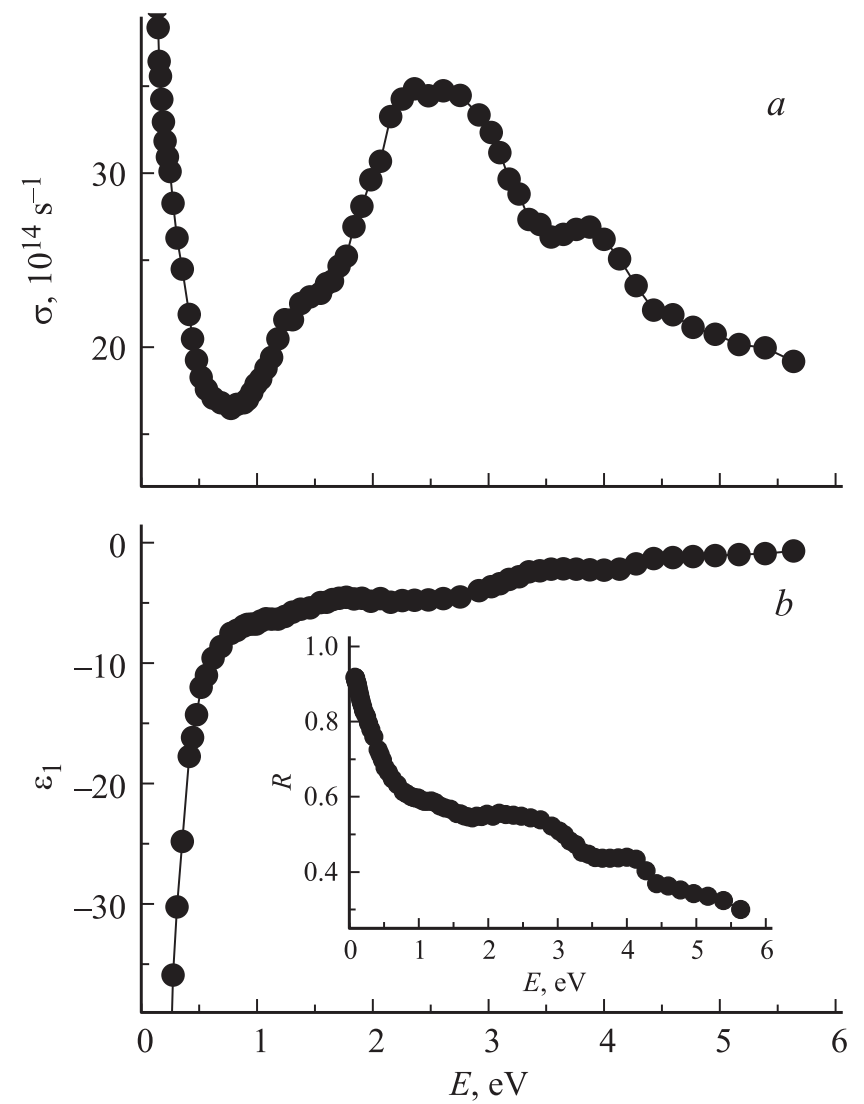

Рис. 3. Дисперсионные зависимости оптической проводимости $(a)$ и действительной части диэлектрической проницаемости $(b)$ соединения $\mathrm{Gd}_{5} \mathrm{Si}_{3}$. На вставке приведена отражательная способность.

ной дисперсности. С увеличением длины волны света, как показывает рисунок, величины оптических постоянных, за исключением коротковолнового интервала до $\sim 1 \mu \mathrm{m}$, монотонно возрастают при сохранении неравенства $k>n$. По значениям $n$ и $k$ рассчитаны действительная $\varepsilon_{1}=n^{2}-k^{2}$ и мнимая $\varepsilon_{2}=2 n k$ части комплексной диэлектрической проницаемости, оптическая проводимость $\sigma=n k c / \lambda(c-$ скорость света), а также отражательная способность $\left.R=\left[(n-1)^{2}+k^{2}\right] /[n+1)^{2}+k^{2}\right]$. Обращает внимание, что энергетические зависимости этих характеристик, представленные на рис. 3, типичны для материалов с металлическим типом проводимости - значения $\varepsilon_{1}$ отрицательны при всех длинах волн, а величина отражательной способности с уменьшением энергии света стремится к единице.

Рассмотрим подробнее спектр оптической проводимости $\sigma(E)$ соединения $\mathrm{Gd}_{5} \mathrm{Si}_{3}$ (рис. 3,a). Данный параметр характеризует частотную зависимость и интенсивность оптического отклика среды и в отличие от статической проводимости определяется не только плотностью состояний на уровне Ферми $N\left(E_{\mathrm{F}}\right)$, но также особенностями ее дисперсии во всем исследуемом интервале. В структуре экспериментального спектра $\sigma(E)$ отчетливо выражены два интервала, соответствующие различным типам взаимодействия электрона с электромагнитной волной. При низких частотах (инфракрасная область) поведение $\sigma(E)$ типично для внутризонного (друдевского) механизма возбуждения свободных электронов квантами света малой энергии $\left(\sigma \sim 1 / \omega^{2}\right)$. Вклад этого механизма определяется параметрами носителей тока [31] - релаксационной $\gamma$ и плазменной $\omega_{p}$ частотами электронов проводимости. Значения данных параметров, рассчитанных по формулам Друде, равны $\gamma=2.2 \cdot 10^{14} \mathrm{~s}^{-1}$ и $\omega_{p}=4.2 \cdot 10^{15} \mathrm{~s}^{-1}$. Из соотношения $N=\omega_{p}^{2} m / 4 \pi e^{2}$ (m,e- заряд и масса электрона) получаем величину концентрации свободных электронов $N=0.65 \cdot 10^{22} \mathrm{~cm}^{-3}$.

С увеличением энергии фотона (видимый и ультрафиолетовый интервалы) начинает доминировать механизм квантового поглощения света, кардинально изменяющий характер частотной зависимости оптической проводимости. Монотонный низкоэнергетический спад сменяется широкой полосой фундаментального поглощения с двумя максимумами различной интенсивности, расположенными вблизи $2.5 \mathrm{eV}$ и $3.8 \mathrm{eV}$. Структура данной полосы связана с межзонными переходами между электронными состояниями, локализованными по обе стороны от фермиевской границы, и определяется особенностями зонного спектра данного соединения. Для понимания природы формирования межзонного поглощения в данном спектральном интервале представляется интересным сравнить экспериментальную межзонную оптическую проводимость, полученную вычитанием друдевского вклада из опытной $\sigma(E)$, с соответствующей зависимостью, рассчитанной из полной плотности электронных состояний $N(E)$. Для вычисления последней использовался метод [32], в котором теоретическая межзонная проводимость выражается интегральной функцией на основе свертки плотностей состояний выше и ниже $E_{\mathrm{F}}$. При этом общая картина квантового поглощения определяется суперпозицией вкладов от энергетических зон с противоположно направленными спинами.

Такой расчет, реализованный в приближении равной вероятности всех типов квантовых переходов, представлен на рис. 4 в произвольных единицах совместно с экспериментальной кривой. Сравнение показывает, что рассчитанная частотная дисперсия межзонной оптической проводимости качественно объясняет природу квантового поглощения в $\mathrm{Gd}_{5} \mathrm{Si}_{3}$ и проявляет существенное сходство с зависимостью, наблюдаемой в эксперименте. Это относится как к оценкам общей формы кривых и интервала интенсивного поглощения света, так и локализации двух основных максимумов. На рис. 4 также приведены рассчитанные парциальные вклады в межзонную проводимость от переходов, происходящих с вовлечением $4 f$ - и $5 d$-электронных состояний $\mathrm{Gd}$, а также суммарный вклад от переходов с участием Gd 6p- и Si $3 p$-электронов. Отчетливо видно, что преобладающий вклад в формирование главных особенностей оптической проводимости - максимумов при 2.5 и 


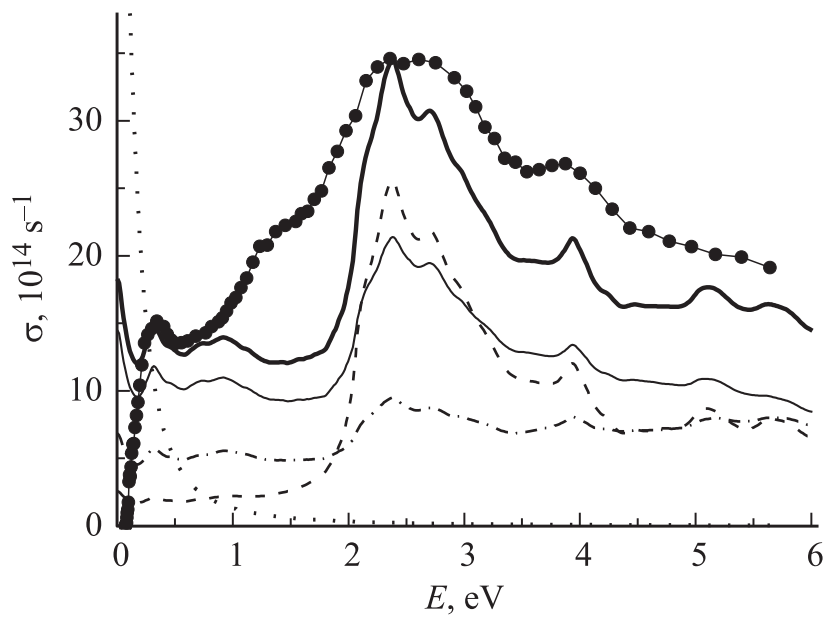

Рис. 4. Спектры межзонной оптической проводимости соединения $\mathrm{Gd}_{5} \mathrm{Si}_{3}$. Точки - эксперимент. Толстая сплошная кривая - расчет из полной плотности состояний, тонкая сплошная и штриховая линии - вклады от переходов с участием $\mathrm{Gd} 5 d$ - и $\mathrm{Gd} 4 f$-электронов. Штрихпунктирная линия - суммарный вклад от переходов с участием Gd $6 p$ и Si $3 p$-электронов. Пунктирная линия - друдевский вклад.

$3.8 \mathrm{eV}$, вносят переходы с участием $4 f$ - и $5 d$-энергетических зон Gd. Анализ структуры рассчитанной плотности состояний (рис. 1) дает возможность идентифицировать эти пики с переходами из 5d-зоны Gd (максимумы вблизи $0.3-0.6 \mathrm{eV}$ и $1.9 \mathrm{eV}$ ниже $\left.E_{\mathrm{F}}\right)$ в $4 f$-зону $\mathrm{Gd}$, центр которой расположен при $2.1 \mathrm{eV}$ выше $E_{\mathrm{F}}$. В низкоэнергетическом интервале ниже $2 \mathrm{eV}$ квантовое поглощение связано с переходами в системе гибридизированных $d$ и $p$-зон $\mathrm{Gd}$ и $\mathrm{Si}$, локализованных по разные стороны от уровня Ферми. Согласно расчету, вклады в $\sigma(E)$ от других типов электронных переходов по величине существенно слабее. В целом следует отметить более сглаженный характер опытной зависимости межзонной оптической проводимости по сравнению с теоретической. К подобному сглаживанию, как правило, приводит экспериментальный фактор, связанный с несовершенством приготовления зеркальной отражающей поверхности. В заключение отметим качественное соответствие полученных в настоящей работе результатов с данными по исследованию рентгеновской и УФ фотоэмиссии $[20,21]$. Сравнение показывает, что рассчитанная плотность электронных состояний и экспериментальная частотная дисперсия оптической проводимости $\mathrm{Gd}_{5} \mathrm{Si}_{3}$ отражают основные особенности структуры валентной зоны, выявленные в фотоэлектронных спектрах данного соединения.

\section{4. Заключение}

Таким образом, в работе впервые были исследованы электронная структура и оптические свойства соединения $\mathrm{Gd}_{5} \mathrm{Si}_{3}$. В спин-поляризованных расчетах, прове- денных методом LSDA + U с учетом сильных взаимодействий в $4 f$-оболочке ионов $\mathrm{Gd}$, рассчитаны полные и парциальные плотности электронных состояний. Эллипсометрическим методом в спектральном интервале $0.22-15 \mu \mathrm{m}$ измерены оптические характеристики данного интерметаллида. На основе результатов расчета проведена интерпретация экспериментальной зависимости межзонной оптической проводимости. Показано, что частотная дисперсия этого параметра удовлетворительно воспроизводится в рамках представленного расчета. Локализация и ширина основных структурных особенностей на опытной кривой оптической проводимости качественно согласуются с соответствующей теоретической зависимостью.

\section{Список литературы}

[1] V.K. Pecharsky, K.A. Gschneidner,jr. Phys. Rev. Lett. 78, 4494 (1997).

[2] L. Morellon, P.A. Algarabel, M.R. Ibarra, J. Blasco, B. García-Landa. Phys. Rev. B 58, R14721 (1998).

[3] L. Morellon, J. Blasco, P.A. Algarabel, M.R. Ibarra. Phys. Rev. B 62, 1022 (2000).

[4] V.K. Pecharsky, A.P. Holm, K.A. Gschneidner,jr., R. Rink. Phys. Rev. Lett. 91, 197204 (2003).

[5] Ya. Mudryk, A.P. Holm, K.A. Gschneidner,jr., V.K. Pecharsky. Phys. Rev. B 72, 06442 (2005).

[6] V.K. Pecharsky, K.A. Gschneidner,jr. In: Magnetism and Structure in Functional Materials / Ed. A. Planes, L. Mañosa, A. Saxena. Springer, Heidelberg (2005). V. 79. 251 p.

[7] W. Choe, V.K. Pecharsky, A.O. Pecharsky, K.A. Gschneidner,jr., V.G. Young,jr., G.J. Miller. Phys. Rev. Lett. 84, 4617 (2000).

[8] Ya. Mudryk, Y. Lee, T. Vogt, K.A. Gschneidner,jr., V.K. Pecharsky. Phys. Rev. B 71, 174104 (2005).

[9] C. Magen, L. Morellon, P.A. Algarabel, M.R. Ibarra, Z. Arnold, J. Kamarad, T.A. Lograsso, D.L. Schlagel, V.K. Pecharsky, A.O. Tsokol, K.A. Gschneidner,jr. Phys. Rev. B 72, 024416 (2005).

[10] P. Boulet, F. Weitzer, K. Hiebl, H. Noël. J. Alloys Comp. 315, 75 (2001).

[11] J.P. Semitelou, J.K. Yakinthos. JMMM 265, 152 (2003).

[12] N. Mohapatra, K. Mukherjee, K.K. Iyer, E.V. Sampathkumaran. J. Phys.: Condens. Matter 23, 496001 (2011).

[13] A.V. Morozkin, O. Isnard, R. Nirmala, S.K. Malik. J. Alloys Comp. 470, 20 (2009).

[14] N. Mohapatra, S.D. Das, K. Mukherjee, K.K. Iyer, E.V. Sampathkumaran. Phys. Rev. B 80, 214425 (2009).

[15] M. Falkowski, A. Kowalczyk, T. Toliński. JMMM 331, 144 (2013).

[16] K.K. Iyer, K. Mukherjee, P.L. Paulose, E.V. Sampathkumaran, Y. Xu, W. Löser. Solid State Commun. 152, 522 (2012).

[17] N.A. Zabidi, H.A. Kassim, K.N. Shrivastava. AIP Conf. Proc. 1325, 198 (2010).

[18] Yu.V. Knyazev, A.V. Lukoyanov, Yu.I. Kuz'min. Physica B 442, 12 (2014).

[19] J. Roger, M. Ben Yahia, V. Babizhetskyy, J. Bauer, S. Cordier, R. Guérin, K. Hiebl, X. Rocquefelte, J.-Y. Saillard, J.-F. Halet. J. Solid State Chem. 179, 2310 (2006). 
[20] L. Braicovich, E. Puppin, I. Lindau, A. Iandelli, G.L. Olcese, A. Palenzona. Phys. Rev. B 41, 3123 (1990).

[21] C. Chemelli, S. Luridiana, M. Sancrotti, L. Braicovich, F. Ciccacci, A. Iandelli, G.L. Olcese, A. Palenzona. Phys. Rev. B 42, 1829 (1990).

[22] J. Roger, V. Babizhetskyy, K. Hiebl, J.-F. Halet, R. Guérin. J. Alloys Comp. 407, 25 (2006).

[23] F. Canepa, S. Cirafici, M. Napoletano. J. Alloys Comp. 335, L1 (2002).

[24] T. Nakayama, K. Kurosaki, Y. Ohishi, H. Muta, S. Yamanaka. J. Jpn Inst. Met. Mater. 78, 225 (2014).

[25] V.I. Anisimov, F. Aryasetiawan, A.I. Lichtenstein. J. Phys.: Condens. Matter 9, 767 (1997).

[26] O.K. Andersen, O. Jepsen. Phys. Rev. Lett. 53, 2571 (1984).

[27] Ya. Mudryk, D. Paudyal, V.K. Pecharsky, K.A. Gschneidner,jr. Phys. Rev. B 85, 014116 (2012).

[28] S. Gupta, K.G. Suresh, A.V. Lukoyanov. J. Mater. Sci. 50, 5723 (2015).

[29] K.S.V.L. Narasimhan, H. Steinfink, E.V. Ganapathy. J. Appl. Phys. 40, 51 (1969).

[30] E.V. Ganapathy, K. Kugimiya, H. Steinfink, D.I. Tchernev. J. Less-Common Met. 44, 245 (1976).

[31] А.В. Соколов. Оптические свойства металлов. Физматгиз, M. (1961). 464 c

[32] C.N. Berglund, W.E. Spicer. Phys. Rev. 136, A1044 (1964). 